\title{
ARTICLE
}

Clinical Study

\section{Phase 1 study of the ATR inhibitor berzosertib in combination with cisplatin in patients with advanced solid tumours}

\author{
Geoffrey I. Shapiro ${ }^{1}{ }^{1}$, Robert Wesolowski ${ }^{2}$, Craig Devoe ${ }^{3}$, Simon Lord (D) $^{4}$, John Pollard ${ }^{5,11}$, Bart S. Hendriks ${ }^{6,12}$, Martin Falk ${ }^{7,13}$, \\ Ivan Diaz-Padilla ${ }^{8,14}$, Ruth Plummer ${ }^{9}$ and Timothy A. Yap ${ }^{10,15}$
}

\begin{abstract}
BACKGROUND: Berzosertib (formerly M6620, VX-970) is a highly potent and selective, first-in-class ataxia telangiectasia-mutated and Rad3-related protein kinase (ATR) inhibitor. We assessed the safety, tolerability, pharmacokinetics, and preliminary efficacy of berzosertib plus cisplatin.

METHODS: Adult patients with advanced solid tumours refractory or resistant to standard of care therapies received ascending doses of cisplatin (day 1) and berzosertib (days 2 and 9) every 3 weeks (Q3W).

RESULTS: Thirty-one patients received berzosertib $\left(90-210 \mathrm{mg} / \mathrm{m}^{2}\right)$ and cisplatin $\left(40-75 \mathrm{mg} / \mathrm{m}^{2}\right)$ across seven dose levels. The most common grade $\geq 3$ treatment-emergent adverse events were neutropenia (20.0\%) and anaemia (16.7\%). There were two doselimiting toxicities: a grade 3 hypersensitivity reaction and a grade 3 increase in alanine aminotransferase. Berzosertib $140 \mathrm{mg} / \mathrm{m}^{2}$ (days 2 and 9) and cisplatin $75 \mathrm{mg} / \mathrm{m}^{2}$ (day 1) Q3W was determined as the recommended Phase 2 dose. Cisplatin had no apparent effect on berzosertib pharmacokinetics. Of the 31 patients, four achieved a partial response (two confirmed and two unconfirmed) despite having previously experienced disease progression following platinum-based chemotherapy.

CONCLUSIONS: Berzosertib plus cisplatin is well tolerated and shows preliminary clinical activity in patients with advanced solid tumours, warranting further evaluation in a Phase 2 setting.

CLINICAL TRIALS IDENTIFIER: NCT02157792.
\end{abstract}

British Journal of Cancer (2021) 125:520-527; https://doi.org/10.1038/s41416-021-01406-w

\section{BACKGROUND}

The standard of care therapies for the treatment of many tumour types include DNA-damaging chemotherapy agents. ${ }^{1}$ Although chemotherapy may be initially effective, the development of drug resistance is common. ${ }^{2}$ There is an unmet need across different cancers for novel treatments that overcome chemotherapy resistance and improve clinical outcomes.

Ataxia-telangiectasia-mutated (ATM) and Rad3-related protein kinases (ATR) play critical roles in the DNA-damage response (DDR), and regulate cell cycle checkpoint control and the repair of damaged DNA by homologous recombination. ${ }^{3}$ To mediate replication fork stabilisation, ATR is recruited to regions of exposed single-stranded DNA, commonly formed at stalled replication forks resulting from replication stress. ATM is recruited to DNA double-strand breaks that can result from collapsed forks. ${ }^{4}$
Many chemotherapy drugs, including cisplatin, work by inducing potentially lethal DNA damage in cancer cells, although their efficacy is compromised by the efficient repair of DNA damage mediated through the DDR pathway. ${ }^{5}$ Cancer cells harbouring ATM-p53 signalling pathway defects are increasingly reliant on ATR to mitigate DNA damage. ${ }^{6}$ Consistently, preclinical data show a potential lethal sensitivity to ATR inhibition in the presence of DNA damage induced by chemotherapeutic agents, which can ultimately lead to cell death via synthetic lethality. ${ }^{7-10}$ In contrast, non-cancer cells can tolerate the effects of ATR inhibition as a result of intact compensatory ATM signalling. Consequently, ATR inhibition may reduce the DDR capabilities of cancer cells, thereby sensitising them to chemotherapy-induced DNA damage. ${ }^{11}$

Berzosertib (formerly M6620, VX-970) is an intravenous (i.v.), highly potent $\left(I C_{50}=19 \mathrm{nM}\right)$, and selective, first-in-class ATR inhibitor. ${ }^{12}$

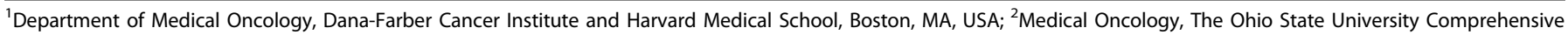

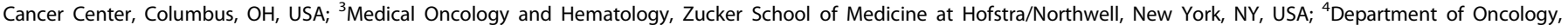

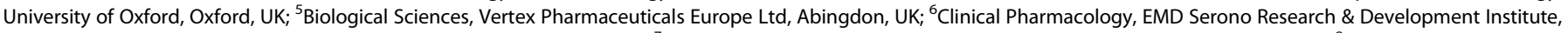

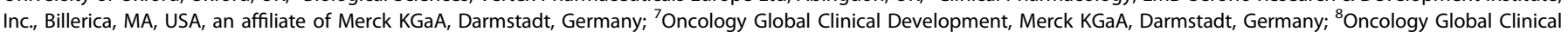

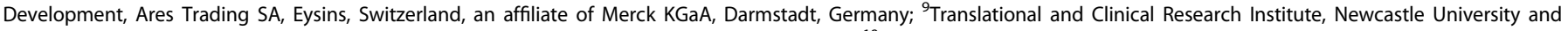

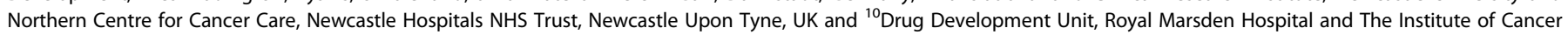
Research, London, UK

Correspondence: Geoffrey I. Shapiro (geoffrey_shapiro@dfci.harvard.edu)

${ }^{11}$ Present address: Bayer plc, Reading, UK

${ }^{12}$ Present address: Novartis Institutes for BioMedical Research, Cambridge, MA, USA

${ }^{13}$ Present address: CureVac, Tübingen, Germany

${ }^{14}$ Present address: GlaxoSmithKline, Zug, Switzerland

${ }^{15}$ Present address: University of Texas MD Anderson Cancer Center, Houston, TX, USA

Received: 5 November 2020 Revised: 22 March 2021 Accepted: 15 April 2021

Published online: 26 May 2021 
Preclinical studies of berzosertib have shown synergy in combination with cisplatin, which led to anti-tumour responses in patient-derived lung cancer xenografts that were otherwise insensitive to cisplatin monotherapy. ${ }^{12}$ The timing of berzosertib administration relative to chemotherapy is critical as preclinical investigations have shown that optimal efficacy is achieved by administering berzosertib $12-24 \mathrm{~h}$ after cisplatin. ${ }^{13}$

The purpose of this first-in-human, open-label, Phase 1 trial (ClinicalTrials.gov, identifier: NCT02157792) was to evaluate the safety, tolerability, pharmacokinetics (PKs), and preliminary antitumour activity of berzosertib in combination with cisplatin.

\section{METHODS}

Study design and treatment

This trial was part of a multicentre, open-label, non-randomised, Phase 1 study separated into six parts (A, B, B2, C1, C2, and C3) (Fig. S1). The focus of this manuscript is part $B$ : berzosertib in combination with cisplatin; the other parts will be reported elsewhere. Part B was a single-arm, $3+3$ dose-escalation Phase 1 study evaluating the safety, tolerability and PK of berzosertib in combination with cisplatin in patients with advanced solid tumours. The study was conducted across two sites in the UK and three sites in the USA between July 2014 (first patient enrolled) and April 2017 (last patient discontinued treatment).

The starting dose of berzosertib was based on the emerging safety data from the ongoing part A study. ${ }^{14}$ Upon initiation of the part $B$ study reported here, patients received berzosertib at what was then the highest dose tolerated in part A (or up to one dose level below). Following screening and baseline evaluations, patients received berzosertib i.v. $\left(90 \mathrm{mg} / \mathrm{m}^{2}\right.$; days 2 and 9) and cisplatin $\left(40 \mathrm{mg} / \mathrm{m}^{2}\right.$; day 1$)$, administered in 21-day cycles. Based on safety data from two concurrent studies, ${ }^{14,15}$ a 7- to 14-day lead-in period with single doses of berzosertib $\left(140 \mathrm{mg} / \mathrm{m}^{2}\right)$ alone was also planned.

For the initial dose escalation, the dose of cisplatin was held constant, while the dose of berzosertib was escalated by a maximum of $50 \%$ in each cohort. After the berzosertib dose was escalated, the dose of cisplatin could be increased in order to find the maximum-tolerated dose (MTD) of berzosertib in combination with cisplatin; the MTD was defined as the highest dose of berzosertib tolerated in combination with a cisplatin dose between 60 and $100 \mathrm{mg} / \mathrm{m}^{2}$, inclusive. Safety data obtained through day 21 of cycle 1, including treatment-emergent adverse events (TEAE), serious TEAEs, laboratory values, electrocardiogram (ECG) results, and available PK data, were analysed to determine the next dose level. Patients received berzosertib with cisplatin until progressive disease (PD) or unacceptable toxicity. Patients who did not experience PD after cycle 4 were eligible to receive additional treatment cycles.

\section{Patients}

Eligible patients were adults aged $\geq 18$ years with histologically or cytologically confirmed advanced solid tumours that were metastatic or unresectable and for which standard curative or palliative measures did not exist or were no longer effective, or for whom regimens containing cisplatin might be considered. Eligible patients had a World Health Organization performance status 0 or 1 ; measurable disease defined by Response Evaluation Criteria in Solid Tumours (RECIST) version 1.1.16 adequate bone marrow, liver, and kidney function; and life expectancy of $\geq 12$ weeks.

Key exclusion criteria included radiotherapy (except palliative), endocrine therapy, immunotherapy or chemotherapy within the 4 weeks prior to receiving study therapy; $>6$ cycles of prior treatment with cisplatin; ongoing toxicity or recent major surgery ( $\leq 2$ weeks of first dose of study drug); active central nervous system (CNS) disease or symptoms within 4 weeks prior to treatment; cardiac conditions within 6 months prior to treatment; prior bone marrow transplant or radiation to $>15 \%$ of bone marrow; and receiving medications that are known to be strong inhibitors or inducers of CYP3A4 that could not be discontinued at least 1 week before the start of treatment and for the duration of the study.

Full inclusion and exclusion criteria are shown in the Supplementary information.

Study assessments and endpoints

The primary objective of the study was to assess the safety and tolerability of multiple ascending doses of i.v. berzosertib in combination with cisplatin, in patients with advanced solid tumours. The secondary objectives were to determine the MTD, PK, and preliminary anti-tumour activity of berzosertib in combination with cisplatin.

Safety evaluations (primary endpoint) included continuous assessment of TEAEs, which were graded using National Cancer Institute Common Terminology Criteria for Adverse Events version 4.0. TEAEs were assessed from the first dose to 14 days ( \pm 7 days) after the last dose of study therapy, while specific dose-limiting toxicity (DLT) events considered related/possibly related to the study drug were recorded up until the end of treatment cycle 1. Safety was evaluated throughout treatment and was used to inform dose-escalation decisions. DLTs were generally defined as any grade $\geq 3$ haematologic or organ toxicity and any cardiac abnormality. Patients were eligible for DLT analysis if they had received all doses of berzosertib and cisplatin in cycle 1 . The full definition of a DLT is shown in Supplementary information. The MTD was defined as the highest dose of berzosertib tolerated in combination with a cisplatin dose between 60 and $100 \mathrm{mg} / \mathrm{m}^{2}$, inclusive.

In cycle 1, plasma samples for PK analysis were collected both pre- and post-dose on days $2-5$ (pre-dose, $0.5 \mathrm{~h}$ before the end of infusion, at the end of infusion, and $0.5,1,2,3,7,23,47$, and $71 \mathrm{~h}$ after end of infusion) and day 9 (pre-dose). Plasma samples were also collected on day 2 of cycle 2 (pre-dose and $2 \mathrm{~h}$ after the end of infusion). Cumulative urine was also collected for PK assessments on days 2 and 3 of cycle 1 (pre-dose to $3,3-7,7-11$, and 11-23 h). Berzosertib concentrations were quantified using a validated liquid chromatography tandemmass spectrometry method and plasma PK were characterised by non-compartmental analyses using Phoenix WinNolin 6.4 (Certara USA Inc., Princeton, NJ, USA).

To assess tumour response, radiological restaging scans (computed tomography, magnetic resonance imaging, or bone scans) were performed at baseline, at the end of cycles 2 and 4, and at the end of every second or third cycle thereafter. Responses were assessed by the investigator according to RECIST version 1.1.

\section{Statistical analysis}

Planned enrolment was $\sim 30$ patients. Sample size and power were based on a standard $3+3$ dose-escalation rule using a binomial model. The maximally tolerated probability of toxicity associated with the dose selected by the standard $3+3$ dose-escalation rule was calculated to range from $\sim 17-26 \%$, with an upper bound of $33 \%$.

The safety set was defined as all patients who received at least one dose of study drug; patients within the safety set were further classified into those who received at least one dose of the study drug in the lead-in period (lead-in safety set) and those who received at least one dose during the combination period (combination safety set). PK data were collected in the PK analysis set, defined as all enrolled patients who received at least one dose of berzosertib and provided at least one measurable post-dose sample. For efficacy analyses, the full analysis set was used, comprising all enrolled patients who had received a baseline scan, 
at least one dose of study drug, and had at least one post-baseline disease assessment.

Standard non-compartmental methods were used to determine PK parameters. Continuous variables were summarised using descriptive summary statistics. Categorical variables (e.g. incidence of a TEAE) were summarised using frequency counts and percentages. TEAEs observed were summarised by system organ class and preferred term according to the Medical Dictionary for Regulatory Activities v.18.0 or higher.

\section{RESULTS}

Patient demographics and disposition

Baseline and disease history characteristics of all 31 patients enrolled are presented in Table 1. The median age was 64.0 years (28-79); $45.2 \%$ were male $(n=14)$. The majority of patients had received prior platinum-based chemotherapy $(n=22)$.

Patient disposition is summarised in Fig. 1. All 31 patients received at least one dose of berzosertib. Part $B$ initially included a 7- to 14-day lead-in period with berzosertib $140 \mathrm{mg} / \mathrm{m}^{2}$ monotherapy $(n=4)$. The lead-in period was later removed from the protocol based on available safety data from this study and a concurrent clinical study. Four patients received berzosertib 140 $\mathrm{mg} / \mathrm{m}^{2}$ alone in the lead-in period for 15 days. One of these patients had PD during the lead-in period and therefore did not receive cisplatin. The other three patients who received berzosertib $140 \mathrm{mg} / \mathrm{m}^{2}$ monotherapy in the lead-in period went on to receive combination therapy.

In the combination period, the majority of patients discontinued treatment with berzosertib and cisplatin due to PD (21 patients $[67.7 \%])$. The median duration of treatment with berzosertib + cisplatin was 44 days (range 2-519).

\section{Dose escalation and DLTs}

There were no DLTs during the monotherapy lead-in period. In the combination period, 30 patients were enrolled across the following six dose levels: berzosertib $90 \mathrm{mg} / \mathrm{m}^{2}$ in combination with cisplatin $40 \mathrm{mg} / \mathrm{m}^{2}(n=3)$; berzosertib $140 \mathrm{mg} / \mathrm{m}^{2}$ in combination with cisplatin $40 \mathrm{mg} / \mathrm{m}^{2}(n=3)$; berzosertib $210 \mathrm{mg} / \mathrm{m}^{2}$ in combination with cisplatin $40 \mathrm{mg} / \mathrm{m}^{2}(n=4)$; berzosertib $210 \mathrm{mg} / \mathrm{m}^{2}$ in combination with cisplatin $60 \mathrm{mg} / \mathrm{m}^{2} \quad(n=10)$; berzosertib $140 \mathrm{mg} / \mathrm{m}^{2}$ in combination with cisplatin $75 \mathrm{mg} / \mathrm{m}^{2}(n=7)$; and berzosertib $210 \mathrm{mg} / \mathrm{m}^{2}$ in combination with cisplatin $75 \mathrm{mg} / \mathrm{m}^{2}(n=3)$ (Fig. 1).

Two DLTs occurred in two different patients while receiving the combination. One patient treated with berzosertib $210 \mathrm{mg} / \mathrm{m}^{2}$ in combination with cisplatin $60 \mathrm{mg} / \mathrm{m}^{2}$ experienced a DLT of grade 3 increase in ALT in the setting of hypotension and ischaemic colitis. The DLT was considered related to cisplatin and occurred on day 3 of cycle 1 . The patient discontinued treatment and the DLT was resolved after $\sim 2$ weeks following treatment with antibiotics and mucolytic agents. The other DLT (grade 3 hypersensitivity reaction) was reported in a patient who received berzosertib $140 \mathrm{mg} / \mathrm{m}^{2}$ in combination with cisplatin $75 \mathrm{mg} / \mathrm{m}^{2}$. Approximately $20 \mathrm{~min}$ into the infusion of berzosertib, the patient became unresponsive for a brief period, developed an erythematous rash (grade 2) on her chest, and had 1 episode of vomiting (grade 1). The DLT was considered related to berzosertib and occurred on day 9 of cycle 1 . The patient discontinued treatment and the DLT was resolved within $24 \mathrm{~h}$ following treatment with a steroid and an antihistamine.

Dose escalation was halted after completing the berzosertib $210 \mathrm{mg} / \mathrm{m}^{2}$ in combination with cisplatin $75 \mathrm{mg} / \mathrm{m}^{2}$ dose level due to chronic haematological toxicities that led to delays in cisplatin dosing. At this dose level, two patients experienced neutropenia (one grade 2 and one grade 3 ). As there was preclinical and clinical evidence of robust target engagement at lower doses, ${ }^{15}$ and as there was good tolerability in the absence of delays in cisplatin dosing, it was decided that berzosertib $140 \mathrm{mg} / \mathrm{m}^{2}$ (days 2 and 9)
Table 1. Patient demographics and baseline characteristics.

\begin{tabular}{|c|c|}
\hline Characteristic & Total $N=31$ \\
\hline \multicolumn{2}{|l|}{ Sex, $n(\%)$} \\
\hline Male & $14(45.2)$ \\
\hline Female & $17(54.8)$ \\
\hline \multicolumn{2}{|l|}{ Race, $n$ (\%) } \\
\hline White & $27(87.1)$ \\
\hline Black or African American & $2(6.5)$ \\
\hline Asian & $2(6.5)$ \\
\hline Median (range) age, years & $64.0(28-79)$ \\
\hline$<65$ years & $16(51.6 \%)$ \\
\hline$\geq 65$ years & $15(48.4 \%)$ \\
\hline \multicolumn{2}{|l|}{ WHO PS, $n(\%)$} \\
\hline 0 & $9(29.0)$ \\
\hline 1 & $22(71.0)$ \\
\hline Prior chemotherapy, $n$ (\%) & $31(100.0)$ \\
\hline Platinum-based chemotherapy ${ }^{a}$ & $22(72.9)$ \\
\hline Non-platinum-based chemotherapy & $31(100.0)$ \\
\hline \multicolumn{2}{|l|}{$\begin{array}{l}\text { Primary tumour location (tumour type } \\
\text { added where applicable), } n(\%)\end{array}$} \\
\hline Colon/rectum (colorectal) & $5(16.2)$ \\
\hline Breast & $4(12.9)$ \\
\hline Other $^{\mathrm{b}}$ & $4(12.9)$ \\
\hline Ovary & 4 (12.9) \\
\hline Lung $^{c}$ & $3(9.7)$ \\
\hline Pancreas & $3(9.7)$ \\
\hline Bile duct (cholangiocarcinoma) & $2(6.5)$ \\
\hline Eye (melanoma) & $1(3.2)$ \\
\hline Oesophagus (squamous cell carcinoma) & $1(3.2)$ \\
\hline Parotid gland (adenoid cystic carcinoma) & $1(3.2)$ \\
\hline Prostate & $1(3.2)$ \\
\hline Thymus (thymoma) & $1(3.2)$ \\
\hline Uterus (endometrial) & $1(3.2)$ \\
\hline \multicolumn{2}{|c|}{$\begin{array}{l}\text { WHO PS World Health Organization performance status. } \\
\text { a Ten patients received prior cisplatin. } \\
\text { b Included gastrointestinal stromal tumour, liposarcoma of inguinal region, } \\
\text { squamous cell carcinoma, and unknown primary carcinoma. } \\
\text { cIncluded pleural mesothelioma, lung carcinoma, and non-small cell lung } \\
\text { cancer. }\end{array}$} \\
\hline
\end{tabular}

and cisplatin $75 \mathrm{mg} / \mathrm{m}^{2}$ (day 1 ) every 3 weeks (Q3W) would be the recommended Phase 2 dose (RP2D).

Safety

The safety analysis set included 31 patients who received at least one dose of berzosertib, 30 of whom received at least one dose of both berzosertib and cisplatin. An overall summary of TEAEs for berzosertib in combination with cisplatin is shown in Table 2.

Of the patients receiving combination therapy, 29 (96.7\%) had at least one TEAE and 21 (70.0\%) patients had at least one grade $\geq 3$ TEAE (Table 2). The most common grade $\geq 3$ TEAEs were neutropenia (six patients [20.0\%]) and anaemia (five patients [16.7\%]). The most common treatment-related AE of grade $\geq 3$ was neutropenia, which occurred in six $(20.0 \%)$ patients.

A total of 11 (36.7\%) patients had at least one serious TEAE. The most common serious TEAEs were infusion-related reactions, and increases in alanine aminotransferase (ALT) and aspartate aminotransferase (AST), all of which occurred in two patients each. Furthermore, eight (26.7\%) had at least one AE leading to 


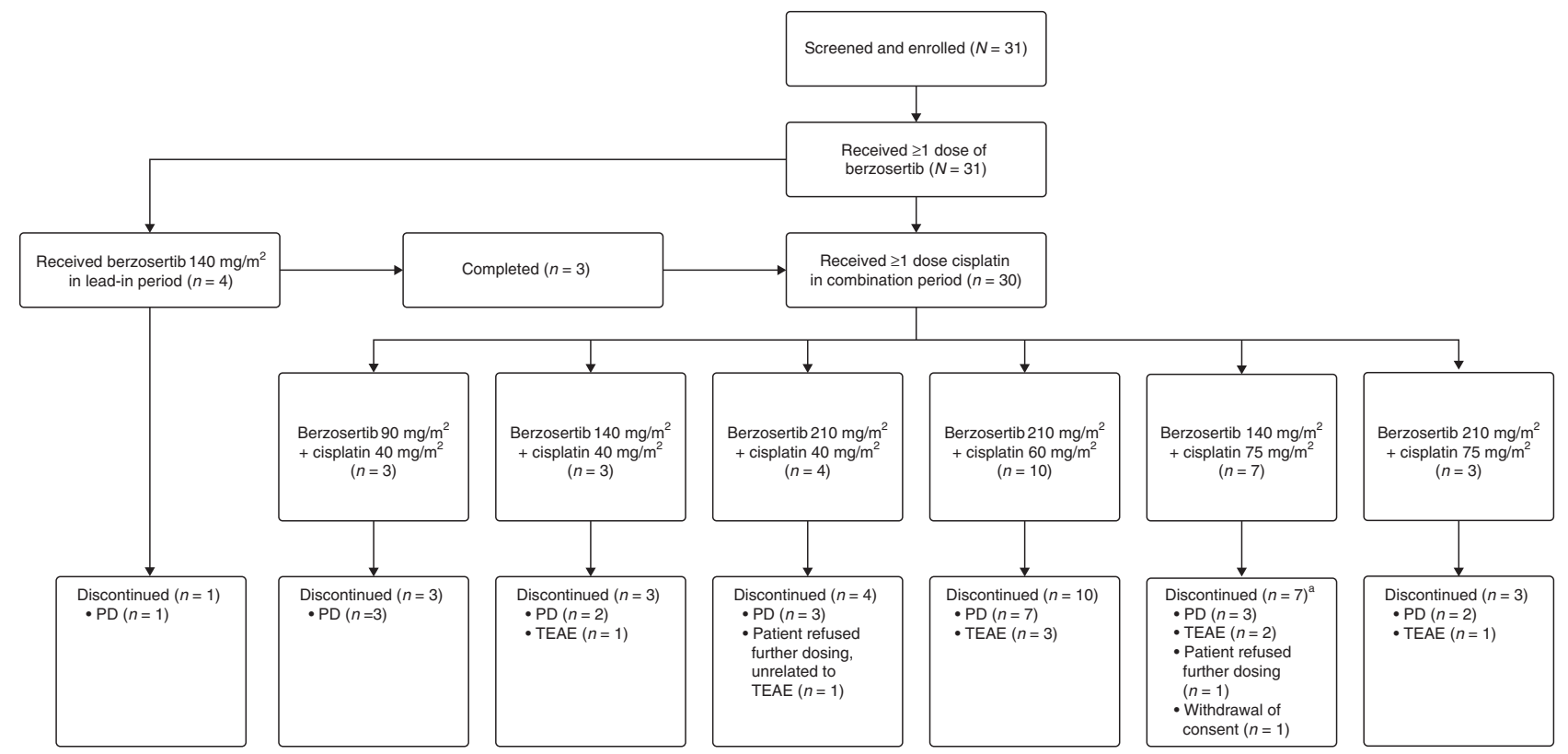

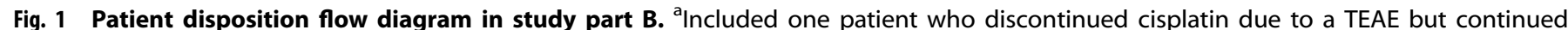
treatment with single-agent berzosertib until disease progression. PD progressive disease, TEAE treatment-emergent adverse event.

treatment discontinuation, including the two patients who experienced serious infusion-related reactions (one related to berzosertib and scored as a DLT, and one related to cisplatin). The patient who discontinued treatment with cisplatin due to a cisplatin-related infusion reaction (hypotension and hypoxia) occurring on day 1 of cycle 13 continued berzosertib monotherapy up to cycle 28 . There were no TEAEs that led to death during the treatment period. The majority of reported deaths were a result of disease-related complications or unconfirmed progression in the palliative care setting.

Finally, there were no clinically meaningful trends attributable to berzosertib treatment identified from laboratory results (serum chemistry, haematology, or urinalysis), vital signs, or ECG parameters.

\section{Pharmacokinetics}

PK assessments for berzosertib were conducted during the monotherapy lead-in period, up to 3 days post administration, days 2-5 and 9 of cycle 1 and day 2 of cycle 2. Plasma PK parameters were determined for the four patients who participated in the monotherapy lead-in period (Table 3). A single data point in the terminal phase of one patient was excluded from PK parameter calculations as it was deemed implausible (10-fold increase from the previous concentration) and strongly skewed the results from the monotherapy lead-in subgroup. In combination with cisplatin, the PK characteristics of berzosertib were determined in 27 patients who received berzosertib $90-210 \mathrm{mg} / \mathrm{m}^{2}$, with profiles shown in Fig. 2 and PK parameters shown in Table 3. The PK characteristics of berzosertib in combination with cisplatin were consistent with the PK of corresponding doses of berzosertib monotherapy determined in part $A$ of this study. ${ }^{14}$ The mean terminal elimination half-life of berzosertib monotherapy was determined in four patients to be $\sim 17 \mathrm{~h}$. Berzosertib plasma exposure was approximately dose proportional, based on the mean plasma concentrations and maximum observed plasma concentration values. The mean renal clearance was 4.7 L/h and the mean percentage of berzosertib excreted in the urine was $6 \%$, indicating that renal clearance constitutes a minor mechanism of berzosertib clearance from the body.

\section{Efficacy}

The full analysis set included 26 (83.9\%) patients who received at least one dose of study drug, had a baseline scan, and had at least one post-baseline disease assessment. Four (15.4\%) patients achieved PR (32.7-100.0\% reduction in tumour lesion diameter) and with the duration of response ranging from 44 to 441 days. Fifteen $(57.7 \%)$ patients achieved SD, one of which lasted for 172 days (Fig. 3 ).

All four patients who achieved PR, including those with advanced high-grade serous ovarian cancer (HGSOC), triple-negative breast cancer (TNBC), castration-resistant prostate cancer with neuroendocrine differentiation, and pleural mesothelioma, had been previously exposed to platinum-based regimens.

The patient with HGSOC was a 75-year-old BRCA2 W2626Q carrier, who had undergone debulking, followed by i.v./intraperitoneal platinum and taxane-based treatment and subsequent carboplatin and doxorubicin, after which she was considered platinum-resistant. She then received paclitaxel and bevacizumab, as well as olaparib without response. She initiated treatment with berzosertib $140 \mathrm{mg} / \mathrm{m}^{2}$ and cisplatin $75 \mathrm{mg} / \mathrm{m}^{2}$. Cisplatin was reduced in cycle 2 to $60 \mathrm{mg} / \mathrm{m}^{2}$ due to grade 4 thrombocytopenia and grade 3 neutropenia during the first cycle. She achieved a confirmed PR and remained on trial for 14 weeks. The reason for treatment discontinuation was a rise in tumour markers, without documented RECIST progression.

The patient with TNBC was a 47-year-old female with a tumour harbouring TP53 R213* mutation and RB1 deletion. After mastectomy, she received radiation and doxorubicin, cyclophosphamide, and paclitaxel in the adjuvant setting. She had recurrent disease after 17 months and was treated with re-excision, gemcitabine and cisplatin; she went on to experience PD after 3 months. She received berzosertib $140 \mathrm{mg} / \mathrm{m}^{2}$ in combination with cisplatin $75 \mathrm{mg} / \mathrm{m}^{2}$. She achieved an unconfirmed PR. After four treatment cycles, new brain and leptomeningeal progression were noted. Outside the CNS, progressive mediastinal adenopathy, and increases in pulmonary, bone, and liver lesions were also observed.

The patient with advanced castration-resistant prostate cancer with neuroendocrine differentiation was a 53-year-old man with a tumour harbouring duplication of exons $1-3$ in $A T R$, as well as $B R C A 2$ and TMPRSS2-ERG somatic tumour mutations. He had previously received androgen deprivation therapy and platinumbased chemotherapy. Following treatment with berzosertib $140 \mathrm{mg} / \mathrm{m}^{2}$ and cisplatin $75 \mathrm{mg} / \mathrm{m}^{2}$, the patient achieved a confirmed PR lasting 69 weeks, with complete regression of liver 
metastases (Fig. 3). ${ }^{17}$ The patient later progressed, developing CNS metastases without evidence of disease progression elsewhere.

Finally, the patient with pleural mesothelioma was a 79-year-old male who had previously achieved SD as his best response following treatment with pemetrexed and cisplatin. He received

Table 2. Overview of TEAEs and TEAEs occurring in $>15 \%$ of patients by preferred term (combination safety set).

\begin{tabular}{|c|c|c|}
\hline \multirow[t]{2}{*}{ Patients, $n$ (\%) } & \multicolumn{2}{|c|}{$\begin{array}{l}\text { Berzosertib + cisplatin } \\
\text { (all doses), } N=30\end{array}$} \\
\hline & Any grade & Grade $\geq 3$ \\
\hline \multicolumn{3}{|l|}{ TEAEs } \\
\hline AEs & $29(96.7)$ & $21(70.0)$ \\
\hline Serious AEs & $11(36.7)$ & $9(30.0)$ \\
\hline \multicolumn{3}{|l|}{ Treatment-related AEs } \\
\hline AEs & $28(93.3)$ & $12(40.0)$ \\
\hline Serious AEs & $3(10.0)$ & $1(3.3)$ \\
\hline AEs leading to study drug discontinuation & $8(26.7)$ & $4(13.3)$ \\
\hline DLTs & & $2(6.7)$ \\
\hline TEAEs occurring in $\geq 15 \%$ of patients & Any grade & Grade $3-4^{a}$ \\
\hline Fatigue & $17(56.7)$ & 0 \\
\hline Anaemia & $15(50.0)$ & $5(16.7)$ \\
\hline Nausea & $15(50.0)$ & $2(6.7)$ \\
\hline Constipation & $11(36.7)$ & 0 \\
\hline Neutropenia & $10(33.3)$ & $6(20.0)$ \\
\hline Vomiting & $9(30.0)$ & 0 \\
\hline Abdominal pain & $7(23.3)$ & $1(3.3)$ \\
\hline Diarrhoea & $7(23.3)$ & 0 \\
\hline Hyponatraemia & $7(23.4)$ & $2(6.7)$ \\
\hline Decreased appetite & $6(20.0)$ & 0 \\
\hline Headache & $6(20.0)$ & $1(3.3)$ \\
\hline Tinnitus & $6(20.0)$ & 0 \\
\hline ALT increased & $5(16.7)$ & $2(6.7)$ \\
\hline AST increased & $5(16.7)$ & $1(3.3)$ \\
\hline Dizziness & $5(16.7)$ & 0 \\
\hline Flushing & $5(16.7)$ & 0 \\
\hline Hypokalaemia & $5(16.7)$ & $1(3.3)$ \\
\hline Hypotension & $4(16.7)$ & $1(3.3)$ \\
\hline
\end{tabular}

$A E$ adverse event, $A L T$ alanine aminotransferase, $A S T$ aspartate aminotransferase, DLT dose-limiting toxicity, TEAE treatment-emergent adverse event.

aNo grade 5 TEAE occurred. five cycles of berzosertib $140 \mathrm{mg} / \mathrm{m}^{2}$ in combination with cisplatin $40 \mathrm{mg} / \mathrm{m}^{2}$ achieving PR, although this could not be confirmed in a subsequent $C T$ scan because the patient discontinued due to grade 1 anorexia (possibly related) and grade 1 chronic kidney failure (unlikely related).

\section{DISCUSSION}

We evaluated the combination of berzosertib and cisplatin as part of the first-in-human trial of berzosertib. The sequential scheduling of chemotherapy with berzosertib was based on extensive preclinical data indicating that ATR inhibition has a maximal impact when administered $12-24 \mathrm{~h}$ after exposure to DNAdamaging agents. Importantly, the collective PK data, including comparison with part A of the study, suggest that preadministration of cisplatin $24 \mathrm{~h}$ before berzosertib administration does not affect the PK profile of berzosertib.

In this portion of the study, the MTD of berzosertib in combination with cisplatin was not reached. Although no protocol-defined DLTs were reported at the highest doses evaluated (berzosertib $210 \mathrm{mg} / \mathrm{m}^{2}$ in combination with cisplatin $75 \mathrm{mg} / \mathrm{m}^{2}$ ), frequent episodes of neutropenia and leukopenia were observed, leading to delays in redosing of cisplatin. The RP2D was, therefore, determined as berzosertib $140 \mathrm{mg} / \mathrm{m}^{2}$ (days 2 and 9) in combination with cisplatin $75 \mathrm{mg} / \mathrm{m}^{2}$ (day 1) Q3W since this dose was generally well tolerated and did not lead to delays in cisplatin dosing. At the RP2D, the safety profile of the combination was generally consistent with that of cisplatin. Importantly, berzosertib exposure at $140 \mathrm{mg} / \mathrm{m}^{2}$ exceeded levels previously shown in preclinical models to result in robust target engagement. In in vivo mouse models, berzosertib $10-20 \mathrm{mg} / \mathrm{kg}$ has demonstrated efficacy in combination with chemotherapy, ${ }^{15,18}$ with the human equivalent dose estimated to be $\sim 60 \mathrm{mg} / \mathrm{m}^{2} .^{19}$ In addition, pharmacodynamic studies performed in the Phase 1 study of berzosertib with carboplatin demonstrated a reduction in serine 345-phosphorylated CHK1 (phospho-CHK1) at doses of berzosertib from $140 \mathrm{mg} / \mathrm{m}^{2} .^{15}$ Therefore, it is likely that the combination achieved a biologically effective dose of berzosertib with full-dose cisplatin, without evidence of PK interactions.

One of the limitations of the current study could arguably be the lack of a comprehensive pharmacodynamic sub-study that would have helped better understand the target engagement and durability of biological effects. It will be important to incorporate such translational evaluation in subsequent berzosertib studies. In addition to a reduction in phospho-CHK1, an increase in phosphoRAD50 has recently been shown to be a promising pharmacodynamic marker of ATR inhibition, resulting from a compensatory increase in ATM activity and therefore applicable in ATM-proficient cells. $^{20}$ At later time points, prolonged $\mathrm{\gamma}-\mathrm{H} 2 \mathrm{AX}$ expression, compared with that achieved after cisplatin alone, may be useful

Table 3. Mean (\%CV) PK parameters of berzosertib in plasma in the lead-in period and for ascending doses of berzosertib in combination with cisplatin (PK analysis set).

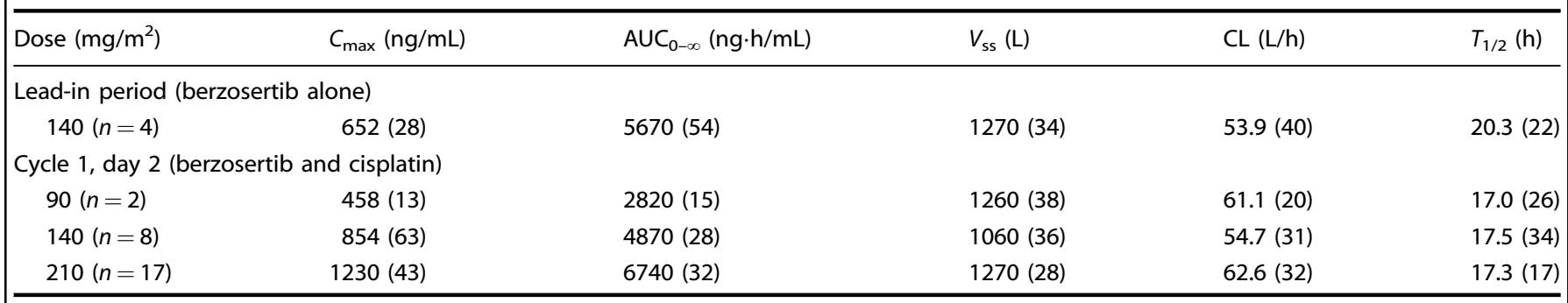

$\mathrm{AUC}_{0-\infty}$ area under the concentration-time curve from the time of dosing extrapolated to infinity, $\mathrm{CL}$ clearance, $C_{\max }$ maximum observed plasma concentration, $C V$ coefficient of variation (in \%), $T_{1 / 2}$ terminal phase half-life, $V_{s s}$ volume of distribution at steady state. 


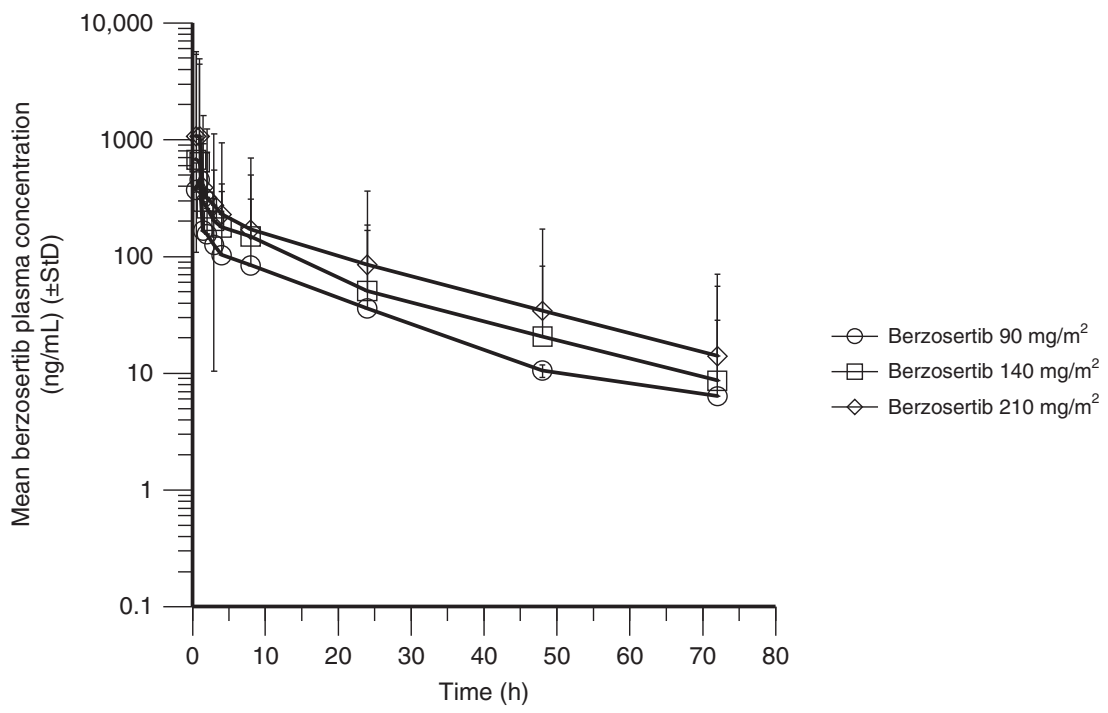

Fig. 2 Plasma concentration-time profile for berzosertib in combination with cisplatin. StD standard deviation.

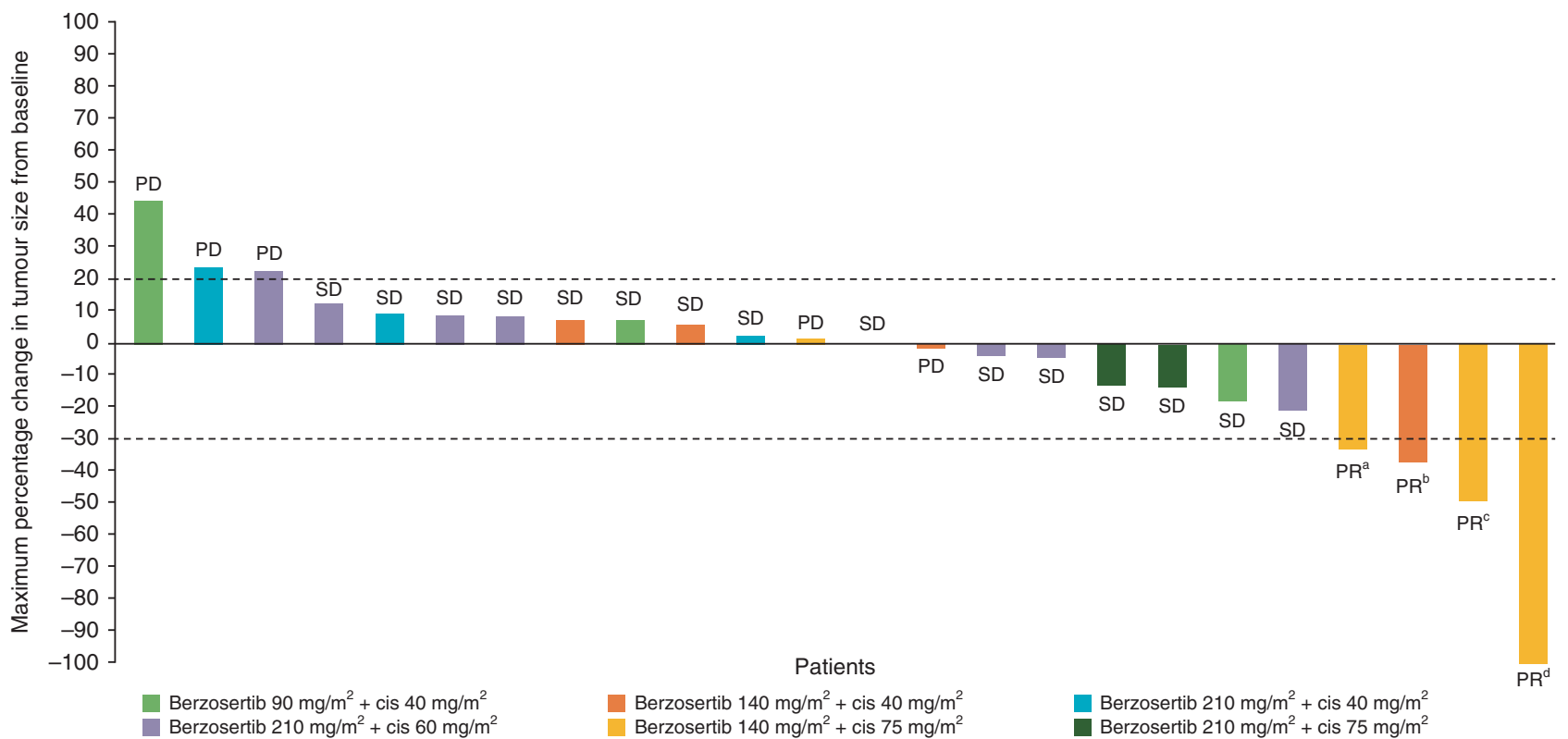

Fig. 3 Maximum percent change in target tumour diameter from baseline. The dashed line at 20\% represents PD, whereas the dashed line

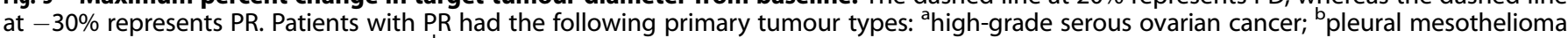
cancer; ${ }^{c}$ triple-negative breast cancer; ${ }^{d}$ castration-resistant prostate cancer. cis cisplatin, PD progressive disease, PR partial response, SD stable disease.

to demonstrate the persistence of DNA damage afforded by the addition of ATR inhibition to chemotherapy. ${ }^{21}$

The majority of patients who received berzosertib in combination with cisplatin achieved disease control with PR or SD as the best response. Of note, PRs were observed in patients who had received prior platinum-based chemotherapy and had experienced disease progression. Notably, the patient with HGSOC and BRCA2 mutation also did not benefit from treatment with olaparib. ATR inhibition can disrupt homologous recombination repair as well as DNA replication fork stability, two major mechanisms of PARP inhibitor resistance. These effects may have contributed to re-sensitising the tumour to cisplatin. ${ }^{22}$

The results of this study add to those of other already reported clinical trials that have evaluated the combination of berzosertib with different chemotherapeutic agents. In a Phase 1 study with berzosertib in combination with topotecan, three of five patients with platinum-resistant small cell lung cancer (SCLC) achieved PR or prolonged SD lasting $\geq 6$ months. ${ }^{23} \mathrm{~A}$ subsequent proof-of-concept Phase 2 study with berzosertib in combination with topotecan in patients with SCLC reported an objective response rate of $36 \%$ $(9 / 25)$, with a median duration of response of 6.4 months, including those with platinum-resistant disease unlikely to respond to topotecan alone. ${ }^{24}$ Furthermore, in a randomised Phase 2 study, patients with platinum-resistant $\mathrm{HGSOC}$, especially those who had a platinum-free interval $<3$ months, experienced longer PFS following treatment with berzosertib in combination with gemcitabine compared to gemcitabine alone. ${ }^{25}$ Taken together, these results may suggest that berzosertib, likely in combination with a synergistic chemotherapeutic drug, such as gemcitabine, a topoisomerase inhibitor or platinum compounds, may be clinically active 
in tumours under replicative stress, such as SCLC or HGSOC, and may potentially help overcome platinum and/or PARP inhibitor resistance.

As continued work with berzosertib ensues, it will be important to consider biomarker-driven approaches that target tumours harbouring ATM truncating mutations or ATM protein loss, TP53 mutations, $B R C A 1$ or $B R C A 2$ mutations, or other alterations conferring homologous recombination repair deficiency. It will also be important to consider genomic changes that are likely to be associated with a high degree of replicative stress, such as CCNE1 or MYC amplification, or RB1 loss. Such tumours may be particularly susceptible to ATR inhibition and may ultimately define populations most likely to benefit from the addition of ATR inhibition to cisplatin or other DNA-damaging agents. The uniform TP53 mutation in TNBC and the TP53 mutation and RB1 loss in SCLC have shaped the study design of the expansion phase of this study (parts C1, C2, and C3). Ultimately, the ability to combine biologically active doses of berzosertib with full doses of chemotherapy portends well for the continued leveraging of ATR inhibition to maximise cytotoxic responses.

In conclusion, berzosertib in combination with cisplatin was well tolerated and showed preliminary signs of efficacy. Further investigations, especially in the PARP inhibitor- and platinumresistant setting, are warranted.

\section{ACKNOWLEDGEMENTS}

We would like to thank patients, investigators, co-investigators, and the study teams at each of the participating centres and at Merck KGaA, Darmstadt, Germany. The UK sites all received funding from Cancer Research UK and the National Institute for Health Research, as Experimental Cancer Medicine Centres. We thank Vertex Pharmaceuticals for their involvement in the development of berzosertib.

\section{AUTHOR CONTRIBUTIONS}

G.I.S., R.W., C.D., S.L., B.S.H., M.F., R.P., and T.A.Y.: acquisition of data. G.I.S., R.W., J.P., B.S.H., M.F., I.D.-P., R.P., and T.A.Y.: analysis and interpretation of data. G.I.S., R.W., S.L., R.P., and T.A.Y.: study supervision. J.P. and R.P.: conception and design. R.P.: development of methodology. M.F.: administrative, technical, or material support. G.I.S., R.W., C.D., S.L., J.P., B.S.H., M.F., I.D.-P., R.P., and T.A.Y.: writing, review, and/or revision of the manuscript. G.I.S., R.W., C.D., S.L., J.P., B.S.H., M.F., I.D.-P., R.P., and T.A.Y.: read and approved the final paper.

\section{ADDITIONAL INFORMATION}

Ethics approval and consent to participate The study was conducted in accordance with the ethical principles of the International Council for Harmonization guideline for Good Clinical Practice and the Declaration of Helsinki, and with applicable local regulations. All patients provided written informed consent before any study procedures were performed. The study sites received ethical approval from the following institutional review boards/independent ethical committees: NRES Committee North East-Sunderland, London-Surrey Borders Research Ethics Committee, Institutional Review Board/Dana Farber Cancer Institute, Western Institutional Review Board, and Biomedical Research Alliance of New York, LLC/Institutional Review Board.

\section{Consent to publish Not applicable.}

Data availability Any requests for data by qualified scientific and medical researchers for legitimate research purposes will be subject to the Merck KGaA, Darmstadt, Germany Data Sharing Policy. All requests should be submitted in writing to the Merck KGaA, Darmstadt, Germany data-sharing portal (https://www. merckgroup.com/en/research/our-approach-to-research-and-development/healthcare/ clinicaltrials/commitment-responsible-data-sharing.html). When Merck KGaA, Darmstadt, Germany has a co-research, co-development, or co-marketing or co-promotion agreement, or when the product has been out-licensed, the responsibility for disclosure might be dependent on the agreement between parties. Under these circumstances, Merck KGaA, Darmstadt, Germany will endeavour to gain agreement to share data in response to requests.

Competing interests G.I.S. has received research funding from Eli Lilly, Merck KGaA/ EMD Serono, Merck \& Co., Inc., and Sierra Oncology; and honoraria for serving on advisory boards for Pfizer, Eli Lilly, G1 Therapeutics, Roche, Merck KGaA/EMD Serono, Sierra Oncology, Bicycle Therapeutics, Fusion Pharmaceuticals, Cybrexa Therapeutics, Astex, Almac, Ipsen, Bayer, Angiex, Daiichi Sankyo, Boehringer Ingelheim, ImmunoMet, Artios, and Asana. R.W. has received research funding from Acerta Pharma and AstraZeneca; served on advisory boards for Puma Biotechnology, Pfizer; and served on speakers' bureau for Roche Diagnostics. C.D. reports honoraria for serving on advisory boards on behalf of Pfizer. S.L. reports personal fees from Eisai, Shionogi, and Prosigna, and was previously employed by Pfizer. J.P. was an employee of Vertex Pharmaceuticals Europe Ltd, Abingdon, UK, at the time of manuscript preparation; and holds stock in Vertex Pharmaceuticals Inc. Current address: Bayer plc, Reading, UK. B.S.H. was an employee of EMD Serono Research \& Development Institute, Inc., Billerica, MA, USA, an affiliate of Merck KGaA, Darmstadt, Germany, at the time of manuscript preparation. Current address: Novartis Institutes for BioMedical Research, Cambridge, MA, USA. M.F. was an employee of Merck KGaA, Darmstadt, Germany, at the time of manuscript preparation. Current address: CureVac, Tübingen, Germany. I.D.-P. was an employee of Ares Trading SA, Eysins, Switzerland, an affiliate of Merck $\mathrm{KGaA}$, Darmstadt, Germany, at the time of manuscript preparation. Current address: GlaxoSmithKline, Zug, Switzerland. R.P. reports honoraria for serving on advisory boards on behalf of Vertex and Merck KGaA relating to this compound; reimbursement for her institution for clinical trials costs; consultancy services to Pierre Faber, Bayer, Octimet, Clovis Oncology, Novartis, Karus Therapeutics, Biosceptre, BMS, Cybrexa, Ellipses, CV6 Therapeutics, Astex Therapeutics, and Sanofi Aventis; and research funding from AstraZeneca. T.A.Y. is employed as a Medical Director of the Institute for Applied Cancer Science, which has a commercial interest in DDR and other inhibitors; has received research support from AstraZeneca, Bayer, Clovis, Constellation, Cyteir, Eli Lilly, EMD Serono, Forbius/Formation Biologics, F-Star, GlaxoSmithKline, Genentech, ImmuneSensor, Ipsen, Jounce, Karyopharm, Kyowa, Novartis, Pfizer, Ribon Therapeutics, Regeneron, Sanofi, Seattle Genetics, Tesaro, and Vertex Pharmaceuticals; and has provided consultancy services for Almac, Aduro, AstraZeneca, Atrin, Axiom, Bayer, Calithera, Clovis, Cybrexa, EMD Serono, F-Star, Guidepoint, Ignyta, I-Mab, Jansen, Kyn Therapeutics, Merck, Pfizer, Roche, Seattle Genetics, and Zai Labs.

Funding information Financial support for this study was provided by Merck KGaA, Darmstadt, Germany and Vertex Pharmaceuticals Inc., Boston, MA, USA. Medical writing assistance was provided by David Lester of Bioscript Stirling Ltd, Macclesfield, UK, and funded by Merck KGaA, Darmstadt, Germany.

Supplementary information The online version contains supplementary material available at https://doi.org/10.1038/s41416-021-01406-w.

Publisher's note Springer Nature remains neutral with regard to jurisdictional claims in published maps and institutional affiliations.

\section{REFERENCES}

1. Rabik, C. A. \& Dolan, M. E. Molecular mechanisms of resistance and toxicity associated with platinating agents. Cancer Treat. Rev. 33, 9-23 (2007).

2. Housman, G., Byler, S., Heerboth, S., Lapinska, K., Longacre, M., Snyder, N. et al. Drug resistance in cancer: an overview. Cancers 6, 1769-1792 (2014).

3. Blackford, A. N. \& Jackson, S. P. ATM, ATR, and DNA-PK: the trinity at the heart of the DNA damage response. Mol. Cell 66, 801-817 (2017).

4. Zeman, M. K. \& Cimprich, K. A. Causes and consequences of replication stress. Nat. Cell Biol. 16, 2-9 (2014).

5. Herzog, T. J. \& Monk, B. J. Bringing new medicines to women with epithelial ovarian cancer: what is the unmet medical need? Gynecol. Oncol. Res Pract. 4, 13 (2017).

6. Kim, D., Liu, Y., Oberly, S., Freire, R. \& Smolka, M. B. ATR-mediated proteome remodeling is a major determinant of homologous recombination capacity in cancer cells. Nucleic Acids Res. 46, 8311-8325 (2018).

7. Wengner, A. M., Siemeister, G., Lücking, U., Lefranc, J., Wortmann, L., Lienau, P. et al. The novel ATR inhibitor BAY 1895344 is efficacious as monotherapy and combined with DNA damage-inducing or repair-compromising therapies in preclinical cancer models. Mol. Cancer Ther. 19, 26-38 (2020).

8. Kim, H. J., Min, A., Im, S. A., Jang, H., Lee, K. H., Lau, A. et al. Anti-tumor activity of the ATR inhibitor AZD6738 in HER2 positive breast cancer cells. Int. J. Cancer 140, 109-119 (2017).

9. Reaper, P. M., Griffiths, M. R., Long, J. M., Charrier, J. D., Maccormick, S., Charlton, P. A. et al. Selective killing of ATM- or p53-deficient cancer cells through inhibition of ATR. Nat. Chem. Biol. 7, 428-430 (2011).

10. Vendetti, F. P., Lau, A., Schamus, S., Conrads, T. P., O'Connor, M. J. \& Bakkenist, C. J. The orally active and bioavailable ATR kinase inhibitor AZD6738 potentiates the 
anti-tumor effects of cisplatin to resolve ATM-deficient non-small cell lung cancer in vivo. Oncotarget 6, 44289-44305 (2015).

11. Minchom, A., Aversa, C. \& Lopez, J. Dancing with the DNA damage response: next-generation anti-cancer therapeutic strategies. Ther. Adv. Med. Oncol. 10, 1758835918786658 (2018).

12. Hall, A. B., Newsome, D., Wang, Y., Boucher, D. M., Eustace, B., Gu, Y. et al. Potentiation of tumor responses to DNA damaging therapy by the selective ATR inhibitor VX-970. Oncotarget 5, 5674-5685 (2014).

13. Pollard, J., Reaper, P., Peek, A., Hughes, S., Gladwell, S., Jones, J. et al. Defining optimal dose schedules for ATR inhibitors in combination with DNA damaging drugs: informing clinical studies of VX-970, the first-inclass ATR inhibitor. Cancer Res. 76, 3717 (2016).

14. Plummer, E. R., Dean, E. J., Evans, T. R. J., Greystoke, A., Herbschleb, K., Ranson, M. et al. Phase I trial of first-in-class ATR inhibitor VX-970 in combination with gemcitabine (Gem) in advanced solid tumors (NCT02157792). J. Clin. Oncol. 34 2513-2513 (2016).

15. Yap, T. A., O'Carrigan, B., Penney, M. S., Lim, J. S., Brown, J. S., de Miguel Luken, M. J. et al. Phase I trial of first-in-class ATR inhibitor M6620 (VX-970) as monotherapy or in combination with carboplatin in patients with advanced solid tumors. J. Clin. Oncol. 38, 3195-3204 (2020).

16. Eisenhauer, E. A., Therasse, P., Bogaerts, J., Schwartz, L. H., Sargent, D., Ford, R. et al. New response evaluation criteria in solid tumours: revised RECIST guideline (version 1.1). Eur. J. Cancer 45, 228-247 (2009).

17. Saito, Y. D., Li, Z., Lustberg, M., Grenade, C. \& Wesolowski, R. Remarkable response to a novel ATR inhibitor in a patient with poorly differentiated neuroendocrine carcinoma. Cancer Treat. Res. Commun. 16, 9-12 (2018).

18. Kurmasheva, R. T., Kurmashev, D., Reynolds, C. P., Kang, M., Wu, J., Houghton, P. J. et al. Initial testing (stage 1) of M6620 (formerly VX-970), a novel ATR inhibitor, alone and combined with cisplatin and melphalan, by the Pediatric Preclinical Testing Program. Pediatr Blood Cancer 65, https://doi.org/10.1002/pbc.26825 (2018).

19. FDA U. Guidance for industry: estimating the maximum safe starting dose in initial clinical trials for therapeutics in adult healthy volunteers. https://www.fda. gov/media/72309/download (2005).

20. Jones, G. N., Rooney, C., Griffin, N., Roudier, M., Young, L. A., Garcia-Trinidad, A. et al. pRAD50: a novel and clinically applicable pharmacodynamic biomarker of both ATM and ATR inhibition identified using mass spectrometry and immunohistochemistry. Br. J. Cancer 119, 1233-1243 (2018).

21. Redon, C. E., Nakamura, A. J., Zhang, Y. W., Ji, J. J., Bonner, W. M., Kinders, R. J. et al. Histone gammaH2AX and poly(ADP-ribose) as clinical pharmacodynamic biomarkers. Clin. Cancer Res. 16, 4532-4542 (2010).

22. Yazinski, S. A., Comaills, V., Buisson, R., Genois, M. M., Nguyen, H. D., Ho, C. K. et al. ATR inhibition disrupts rewired homologous recombination and fork protection pathways in PARP inhibitor-resistant BRCA-deficient cancer cells. Genes Dev. 31, 318-332 (2017).

23. Thomas, A., Redon, C. E., Sciuto, L., Padiernos, E., Ji, J., Lee, M. J. et al. Phase I study of ATR inhibitor M6620 in combination with topotecan in patients with advanced solid tumors. J. Clin. Oncol. 36, 1594-1602 (2018).

24. Thomas, A., Takahashi, N., Rajapakse, V. N, Zhang, X., Sun, Y., Ceribelli, M. et al. Therapeutic targeting of ATR yields durable regressions in small cell lung cancers with high replication stress. Cancer Cell 39, 566-579.e7 (2021).

25. Konstantinopoulos, P. A., Cheng, S.-C., Wahner Hendrickson, A. E., Penson, R. T., Schumer, S. T., Doyle, L. A. et al. Berzosertib plus gemcitabine versus gemcitabine alone in platinum-resistant high-grade serous ovarian cancer: a multicentre, open-label, randomised, phase 2 trial. Lancet Oncol. 21, 957-968 (2020).

(i) Open Access This article is licensed under a Creative Commons Attribution 4.0 International License, which permits use, sharing, adaptation, distribution and reproduction in any medium or format, as long as you give appropriate credit to the original author(s) and the source, provide a link to the Creative Commons license, and indicate if changes were made. The images or other third party material in this article are included in the article's Creative Commons license, unless indicated otherwise in a credit line to the material. If material is not included in the article's Creative Commons license and your intended use is not permitted by statutory regulation or exceeds the permitted use, you will need to obtain permission directly from the copyright holder. To view a copy of this license, visit http://creativecommons. org/licenses/by/4.0/.

(c) The Author(s) 2021 